\title{
Serum microRNA-125a-5p, a useful biomarker in liver diseases, correlates with disease progression
}

\author{
JIANJIAN ZHENG ${ }^{1}$, ZHENXU ZHOU ${ }^{2}$, ZIQIANG XU ${ }^{3}$, GUOJUN LI $^{4}$, PEIHONG DONG ${ }^{5}$, \\ ZHANGUO CHEN ${ }^{6}$, DEZHAO LIN ${ }^{7}$, BICHENG CHEN $^{1}$ and FUJUN YU ${ }^{5}$ \\ ${ }^{1}$ Wenzhou Key Laboratory of Surgery; ${ }^{2}$ Department of Laparoscopic Surgery; ${ }^{3}$ Institute of Organ Transplantation, \\ The First Affiliated Hospital of Wenzhou Medical University, Wenzhou, Zhejiang 325000; ${ }^{4}$ Department of Hepatology, \\ Ningbo Yinzhou Second Hospital, Ningbo, Zhejiang 315000; ${ }^{5}$ Department of Infectious Diseases; \\ ${ }^{6}$ Centre for Laboratory Diagnosis, The First Affiliated Hospital of Wenzhou Medical University; \\ ${ }^{7}$ Department of General Surgery, Wenzhou Hospital of Traditional Chinese Medicine, Wenzhou, \\ Zhejiang 325000, P.R. China
}

Received June 16, 2014; Accepted March 3, 2015

DOI: $10.3892 / \mathrm{mmr} .2015 .3546$

\begin{abstract}
It has been demonstrated that liver microRNA-125a-5p (miR-125a-5p) is correlated with disease progression in different liver diseases, including liver fibrosis and hepatocellular carcinoma (HCC). The present study investigated whether serum miR-125a-5p correlated with the progression of different liver diseases. Serum samples were obtained from healthy individuals, patients with chronic hepatitis B who had undergone a liver biopsy, and patients with HCC and were analyzed for the levels of miR-125a-5p. Compared with the healthy controls, the serum levels of miR-125a-5p were significantly higher in the liver fibrosis serum, and were reduced in HCC. With the development of liver fibrosis, there was a significant increase in the expression of miR-125a-5p $(\mathrm{P}<0.05)$. In comparing histological activity index (HAI) scores, higher expression levels of miR-125a-5p were observed in the high HAI score group $(\mathrm{P}<0.05)$. Furthermore, correlation between serum miR-125a-5p and viral replication $(\mathrm{P}<0.001)$ was observed. Notably, miR-125a-5p demonstrated significant correlation with other markers in the liver fibrosis group $(\mathrm{P}<0.001)$. In the patients with $\mathrm{HCC}$, lower serum levels of miR-125a-5p were correlated with a poor prognosis, determined by Kaplan-Meier curve analysis $(\mathrm{P}=0.009)$. In the liver
\end{abstract}

Correspondence to: Mr. Fujun Yu, Department of Infectious Diseases, The First Affiliated Hospital of Wenzhou Medical University, 2 Fuxue Lane, Wenzhou, Zhejiang 325000, P.R. China E-mail: tjyufujun@163.com

Mr. Bicheng Chen, Wenzhou Key Laboratory of Surgery, The First Affiliated Hospital of Wenzhou Medical University, 2 Fuxue Lane, Wenzhou, Zhejiang 325000, P.R. China

E-mail: fire717@163.com

Key words: serum microRNA-125a-5p, liver fibrosis, hepatocellular carcinoma, biomarker fibrosis and HCC groups, different expression levels of serum miR-125a-5p were observed, and were correlated with disease progression. The results of the present study suggested that serum miR-125a-5p may be used as a non-invasive biomarker for monitoring disease progression in liver diseases.

\section{Introduction}

MicroRNAs (miRNAs) are a class of small non-coding RNAs, which can downregulate gene expression through binding to the 3'-untranslated region of target mRNAs, resulting in mRNA degradation and translation inhibition (1). They have crucial roles in the control of several physiological processes, including development, differentiation, apoptosis, proliferation and metabolism, however, abnormally expressed miRNAs are involved in various human diseases, including cancer (2). miRNAs located in fragile sites or aberrant genomic regions have oncogenic or suppressive roles in cancer (3). Notably, different expression levels of the same miRNA can occur in various human diseases. For example, the expression levels of miRNA (miR)-125a-5p in liver fibrosis and hepatocellular carcinoma (HCC) may be different $(4,5)$. miR-125a is located at $19 \mathrm{q} 13$, which is frequently deleted in types of human cancer. A previous study demonstrated that the expression of miR-125a-5p was decreased in HCC tissues and cell lines, and was associated with aggressive pathological features (4). However, the results differed in liver fibrosis tissues. Higher levels of miR-125-5p were observed in the liver of patients with a histological activity index (HAI) $>6$ and a fibrosis score $>2$, compared with patients with lower scores (5). In addition, the expression of liver miR-125-5p was lowest at $\mathrm{HAI}=0$, fibrosis score $=0$. Additionally, the liver expression of miR-125-5p correlated with plasma hepatitis B virus (HBV)-DNA. From these previous studies, it is evident that miR-125a-5p is a useful marker in liver diseases.

However, obtaining liver tissue is a significant challenge, as liver biopsy is causes pain to the patients, miRNAs in the tissues are unsuitable as diagnostic markers, therefore, a 
non-invasive marker for liver diseases is required. Previous studies have demonstrated that circulating miRNAs are detectable in the serum and plasma in a form, which is sufficiently stable to serve as a biomarker (6-9). Since the levels of miRNAs in the serum and plasma are markedly correlated, either the serum or plasma can be used for the investigation of blood-based biomarkers (8).

Several investigations have reported the role of miR-125a-5p in liver fibrosis and HCC, however, the significance of serum miR-125a-5p in patients with chronic hepatitis B (CHB) and $\mathrm{HCC}$ remains to be elucidated. HBV chronically infects $>250$ million individuals worldwide and is one of the leading causes of hepatitis, cirrhosis and HCC (10). In China, $7.8 \%$ of the population are $\mathrm{HBV}$ carriers of $\mathrm{HBV}$, which is two-thirds of the total number of carriers worldwide (11). Therefore, as a predominant etiological factor, $\mathrm{HBV}$ infection is a major cause of liver disease in China. With the development of chronic HBV infection, the risk of cirrhosis and HCC is increased. This risk is associated with the level of HBV-DNA and the persistence of HBeAg (12). Therefore, the association between serum levels of miR-125a-5p and different stages of liver diseases, including liver fibrosis and HCC, require further elucidation.

The present study investigated the expression of serum miR-125a-5p in patients with CHB and HCC to evaluate its clinical significance in different liver diseases.

\section{Materials and methods}

Study subjects. Blood samples $(5 \mathrm{ml})$ were obtained from patients attending the First Affiliated Hospital of Wenzhou Medical University (Wenzhou, China) and the Second Yinzhou Hosptial (Ningbo, China) between 2007 and 2011 (Table I) through median cubital vein. The present study comprised a total of 164 healthy controls, with normal liver biochemistry, no history of liver disease or alcohol abuse, and no viral hepatitis, 91 patients with $\mathrm{CHB}$ and 120 patients with HCC. Demographic and clinical information was obtained, and a blood sample was collected from each patient. The patients with $\mathrm{CHB}$ were naïve to nucleos(t)ide analogues and interferon therapy, and exhibited no marker of hepatitis C, hepatitis D or human immunodeficiency virus infection, no history of alcohol intake and no clinical or biochemical signs of liver cirrhosis. The blood sample was obtained for each patient on the day which they underwent a diagnostic liver biopsy. The diagnosis of HCC was based on histopathological assessment (13). For patients with HCC, the blood samples were obtained at the time of initial consultation, prior to definitive surgical intervention and/or adjuvant therapy. The follow-up period was used for calculating HCC patient survival rate. The follow-up period was defined as the time from the date of surgery to the date of patient mortality or the last follow-up point. Follow up was completed on January 1, 2013. Informed consent was obtained from all individuals for the use of their blood samples in the present study. The study was approved by the Ethics Committee of the First Affiliated Hospital of Wenzhou Medical University.

Liver histology. Liver biopsy was performed using a 16-gauge Menghini needle. The samples were fixed in $10 \%$ neutral-buffered formalin (Changfeng, Wenzhou, China), embedded in paraffin (Wenzhou Changfeng Biotechnology Co., Ltd., Wenzhou, China) and stained with $1 \mathrm{ml}$ hematoxylin and eosin (Beyotime Institute of Biotechnology, Haimen, China) for $5 \mathrm{~min}$. The samples from $91 \mathrm{CHB}$ patients were reviewed by experienced hepatopathologists in a blinded manner. The HAI and fibrosis stages $(\mathrm{F} 0=$ no fibrosis-F6=cirrhosis) were assessed, according to the Ishak scoring system (14).

Blood sample processing. The blood samples were centrifuged at 3,400 $\mathrm{x}$ g for $7 \mathrm{~min}$ at room temperature using a micro $17 \mathrm{R}$ centrifuge (Thermo Fisher Scientific, Fair Lawn, NJ, USA), within $4 \mathrm{~h}$ of being obtained. The sera were subsequently transferred into Eppendorf tubes (Eppendorf AG, Hamburg, Germany) and centrifuged at $12,000 \mathrm{xg}$ for $10 \mathrm{~min}$ at $4^{\circ} \mathrm{C}$ to remove the remaining cells. The serum samples were stored at $-80^{\circ} \mathrm{C}$ pending RNA extraction.

Detection of $m i R-125 a-5 p$ by reverse transcription-quantitative polymerase chain reaction ( $R T-q P C R)$. Total RNA was extracted from the serum samples using an miRNeasy Mini kit (Qiagen, Carlsbad, California, USA), according to the manufacturer's instructions. To remove any contaminating DNA in the total RNA, $1 \mu 12 \mathrm{U} / \mu 1$ DNase (Qiagen) was used and the final elution volume was $20 \mu \mathrm{l}$. Prior to the RT reaction, the serum RNA preparations were quantified on a Nanodrop 1000 (Nanodrop, Wilmington, Delaware, USA). The RT reaction was performed in a $20 \mu \mathrm{l}$ reaction volume using a TaqMan MicroRNA Reverse Transcription kit (Applied Biosystems, Foster City, CA, USA). For the synthesis of cDNA, the reaction mixtures were sequentially incubated at $16^{\circ} \mathrm{C}$ for $30 \mathrm{~min}, 42^{\circ} \mathrm{C}$ for $30 \mathrm{~min}$ and $85^{\circ} \mathrm{C}$ for $5 \mathrm{~min}$. The $\mathrm{miR}-125 \mathrm{a}-5 \mathrm{p}$ was then quantified in triplicate by qPCR using TaqMan MicroRNA Assay kits (Applied Biosystems). qPCR was performed in an ABI 7500 Real-Time PCR system (Applied Biosystems), according to the standard TaqMan MicroRNA assay instructions, with the following cycles: $95^{\circ} \mathrm{C}$ for $10 \mathrm{~min}$, followed by 40 cycles of $95^{\circ} \mathrm{C}$ for $15 \mathrm{sec}$ and $60^{\circ} \mathrm{C}$ for $60 \mathrm{sec}$. Each amplification reaction was performed in a final volume of $20 \mu \mathrm{l}$ containing $2 \mu \mathrm{l}$ of the cDNA (100 ng/reaction), $10 \mu \mathrm{l}$ TaqMan 2X Universal PCR Master mix without AmpErase Uracil $\mathrm{N}$-glycosylase, $1 \mu \mathrm{l}$ miRNA-specific TaqMan probes, $2 \mu \mathrm{l}$ 10X primers and $5 \mu \mathrm{l}$ RNase-free water (Assay ID 000448; Applied Biosystems). The cycle threshold $(\mathrm{Ct})$ values were calculated using SDS 2.0.1 software (Applied Biosystems). The relative expression levels of miR-125a-5p were calculated and normalized against that of U6 small nuclear RNA (Applied Biosystems), using the comparative $\Delta \mathrm{Ct}$ method with the equation $2^{-\Delta \Delta \mathrm{Ct}}$, as described previously (15).

Serum hepatitis marker and liver fibrosis marker analysis. For the patients with CHB, the HBV-DNA was quantified using an Artus HBV QS-RGQ kit (Qiagen), with a lower detection limit of $10.2 \mathrm{IU} / \mathrm{ml}$. Analyses of the levels of serum hyaluronic acid (HA), laminin (LN), type III procollagen protein (PCIII) and type IV collagen (IV-C) were performed using a Magnetic Immunity Chemiluminescence assay (Shenzen New Industries Biomedical Engineering Co., Ltd., Shenzhen, China).

Statistical analysis. Statistical analyses were performed using SPSS 13.0 software (SPSS, Inc., Chicago, IL, USA). 
Differences between the different groups were determined using a Mann-Whitney U test. Data are presented as the median (range). $\mathrm{P}<0.05$ was considered to indicate a statistically significant difference. Box plots were constructed, with vertical lines indicating the range and horizontal boundaries of the boxes indicating the first and third quartile. The correlation coefficients (r) were calculated using Spearman's correlation. Survival curves were plotted using the Kaplan-Meier method and were analyzed using the log-rank test.

\section{Results}

Patient population. A total of 375 subjects were recruited into the present study (Table I), comprising 164 healthy individuals, 91 patients with liver fibrosis and 120 patients with HCC. In the present study, HBV was the underlying etiology of liver disease in $100 \%$ of the patients in the liver fibrosis group and $85 \%$ of patients in the HCC group.

Expression levels of serum miR-125a-5p in the different groups. The serum levels of miR-125a-5p were detected in different categories (Table II). The median levels of serum miR-125a-5p were 1.44 in the healthy individuals, 3.66 in the liver fibrosis group and 0.68 in the HCC group. These results demonstrated that the levels of serum miR-125a-5p were markedly increased in the liver fibrosis group and significantly reduced in the HCC group, compared with the controls $(\mathrm{P}<0.05$; Fig. 1).

Expression levels of serum miR-125a-5p and quantitative $H B V-D N A$ in liver fibrosis. The results revealed that the expression levels of miR-125a-5p in the different liver fibrosis stages were higher in the liver fibrosis and HCC groups compared with the healthy controls (Table II). Notably, with the development of liver fibrosis, there were significant increases in the expression levels of miR-125a-5p $(\mathrm{P}<0.05$; Fig. $2 \mathrm{~A})$. In terms of the HAI scores, higher expression levels of miR-125a-5p were observed in the high HAI score patient group $(\mathrm{P}<0.05$; Fig. 2B). However, the levels of HBV-DNA were not correlated with the stages of fibrosis or the HAI scores ( $P>0.05$; Table III; Fig. 3A and B). Furthermore, the present study demonstrated that there was a significant positive correlation between the expression levels of miR-125a-5p and the levels of HBV-DNA $(\mathrm{P}<0.001$; Fig. 4).

Expression levels of serum miR-125a-5p and other markers of liver fibrosis. The expression levels of miR-125a-5p were markedly increased in liver fibrosis, compared with the healthy individuals. In addition, the expression levels of other markers associated with liver fibrosis were analyzed (Table IV). The findings demonstrated that there was a significant positive correlation between the expression levels of miR-125a-5p and the expression levels of HA, LN, PCIII and IV-C $(\mathrm{P}<0.001$; Table V).

Expression levels of serum miR-125a-5p in HCC. The expression levels of serum miR-125a-5p were reduced in the patients with HCC compared with the healthy individuals or the liver fibrosis group $(\mathrm{P}<0.05$; Fig. 1A). In addition, the expression levels of miR-125a-5p in patients with HBsAg (+) HCC were higher compared with the patients with $\mathrm{HBsAg}(-) \mathrm{HCC}$

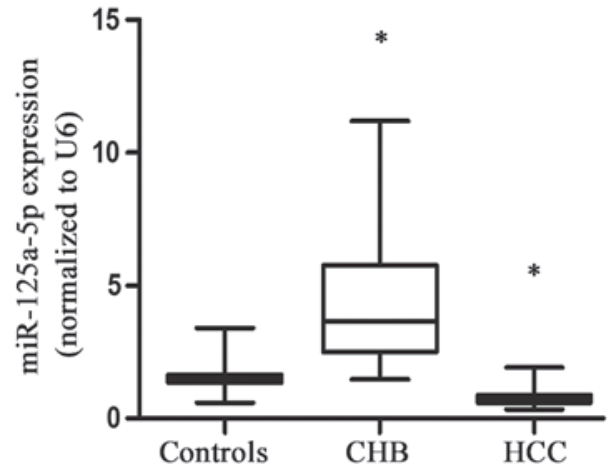

Figure 1. Levels of serum miR-125a-5p were detected in healthy control individuals $(\mathrm{n}=146)$, patients with CHB $(\mathrm{n}=91)$ and HCC $(\mathrm{n}=120) .{ }^{*} \mathrm{P}<0.05$, compared with the healthy controls. Data are presented as the median (range) HCC, hepatocellular carcinoma; $\mathrm{CHB}$, chronic hepatitis $\mathrm{B}$; miR, microRNA. $\mathrm{CHB}$, chronic hepatitis B; HCC, hepatocellular carcinoma.

( $\mathrm{P}<0.05$; Fig. 5). Notably, the expression levels of miR-125a-5p differed between the HCC and liver fibrosis groups, indicating that serum miR-125a-5p may be suitable for use as a diagnostic biomarker in HCC. Additionally, the association between miR-125a-5p and the prognosis in patients with HCC was analyzed by Kaplan-Meier survival analysis. The patients with HCC were divided into low and high expression groups, based on the median expression levels of miR-125a-5p (0.68). The results indicated that patients with HCC exhibiting low expression levels of miR-125a-5p had a lower survival rate compared with those with exhibiting high expression levels of miR-125a-5p, with median survival rates of 30.66 and 39.04 months, respectively; $\mathrm{P}=0.009$; Fig. 6).

\section{Discussion}

In China, $\mathrm{CHB}$ is a significant public health problem, due to the huge number of infected individuals and high costs of treatment (16). Chronic HBV infection induces a series of liver diseases, ranging between a clinically asymptomatic carrier state and the development of cirrhosis-associated complications and HCC $(17,18)$. The predominant goal of clinical antiviral therapy is to inhibit the progression of liver disease. In the present study, significant increases in the expression levels of serum miR-125a-5p were observed in different states of liver fibrosis, between F1 and F6. This indicated that increased serum miR-125a-5p was correlated with disease progression. A similar result was observed in the correlation between expression levels of serum miR-125a-5p and HAI scores. However, no correlation was observed between the levels of HBV-DNA and the stage of liver fibrosis stages, nor was there any correlation between the levels of HBV-DNA and the HAI score. This indicated that the levels of HBV-DNA may not provide a good biomarker for the development of liver fibrosis. However, liver miR-125a-5p, identified as an independent predictor of disease progression by multivariate logistic regression analysis, has been associated with increased severity of disease progression (5). The results of the present study were in accordance with this previous study.

It has been reported that high levels of HBV-DNA can increase the risk of cirrhosis and HCC (12). One of criteria for antiviral therapy is that HBV-DNA levels are 
Table I. Characteristics of the patients enrolled.

\begin{tabular}{|c|c|c|c|c|}
\hline Group & Median age; range (years) & Gender (male/female) & Etiology & Fibrosis stage \\
\hline Healthy control $(n=164)$ & $48 ; 16-81$ & $98 / 66$ & & \\
\hline \multirow[t]{7}{*}{ Liver fibrosis $(\mathrm{n}=91)$} & $49 ; 22-56$ & $52 / 39$ & $\mathrm{HBV}(\mathrm{n}=91)$ & $\mathrm{F} 0(\mathrm{n}=0)$ \\
\hline & & & & $\mathrm{F} 1(\mathrm{n}=15)$ \\
\hline & & & & $\mathrm{F} 2(\mathrm{n}=24)$ \\
\hline & & & & $\mathrm{F} 3(\mathrm{n}=18)$ \\
\hline & & & & $\mathrm{F} 4(\mathrm{n}=21)$ \\
\hline & & & & F5 $(n=3)$ \\
\hline & & & & $\mathrm{F} 6(\mathrm{n}=10)$ \\
\hline $\mathrm{HCC}(\mathrm{n}=120)$ & $60 ; 25-83$ & $75 / 45$ & $\operatorname{HBV}(n=102)$ & \\
\hline
\end{tabular}

HCC, hepatocellular carcinoma; HBV, hepatitis B virus.

Table II. Expression levels of serum miR-125a-5p in different groups.

\begin{tabular}{|c|c|c|c|c|c|c|}
\hline \multirow[b]{2}{*}{ Group } & \multirow[b]{2}{*}{$\mathrm{n}$} & \multirow[b]{2}{*}{ Median } & \multicolumn{2}{|c|}{$\begin{array}{c}\text { Data range } \\
(\mathrm{miR}-125 \mathrm{~A}-5 \mathrm{p} / \mathrm{U} 6)\end{array}$} & \multicolumn{2}{|c|}{$\begin{array}{c}\text { Data range } \\
\text { (miR-125A-5p/U6) }\end{array}$} \\
\hline & & & $25 \%$ & $75 \%$ & $5 \%$ & $95 \%$ \\
\hline Healthy control & 164 & 1.44 & 1.34 & 1.64 & 0.79 & 1.92 \\
\hline Liver fibrosis & 91 & 3.66 & 2.50 & 5.76 & 1.65 & 9.70 \\
\hline $\mathrm{F} 1$ & 15 & 2.14 & 1.93 & 2.32 & 1.56 & 2.62 \\
\hline $\mathrm{F} 2$ & 24 & 2.78 & 2.05 & 3.17 & 1.47 & 4.14 \\
\hline $\mathrm{F} 3$ & 18 & 4.22 & 3.49 & 5.32 & 2.50 & 6.47 \\
\hline F4 & 21 & 4.70 & 3.56 & 6.74 & 2.66 & 8.81 \\
\hline F5 & 3 & 5.47 & 5.34 & 5.87 & 5.34 & 5.87 \\
\hline F6 & 10 & 9.35 & 8.57 & 10.55 & 7.90 & 11.20 \\
\hline $\mathrm{HCC}$ & 120 & 0.68 & 0.60 & 0.88 & 0.46 & 1.42 \\
\hline
\end{tabular}

HCC, hepatocellular carcinoma; F, fibrosis stage.
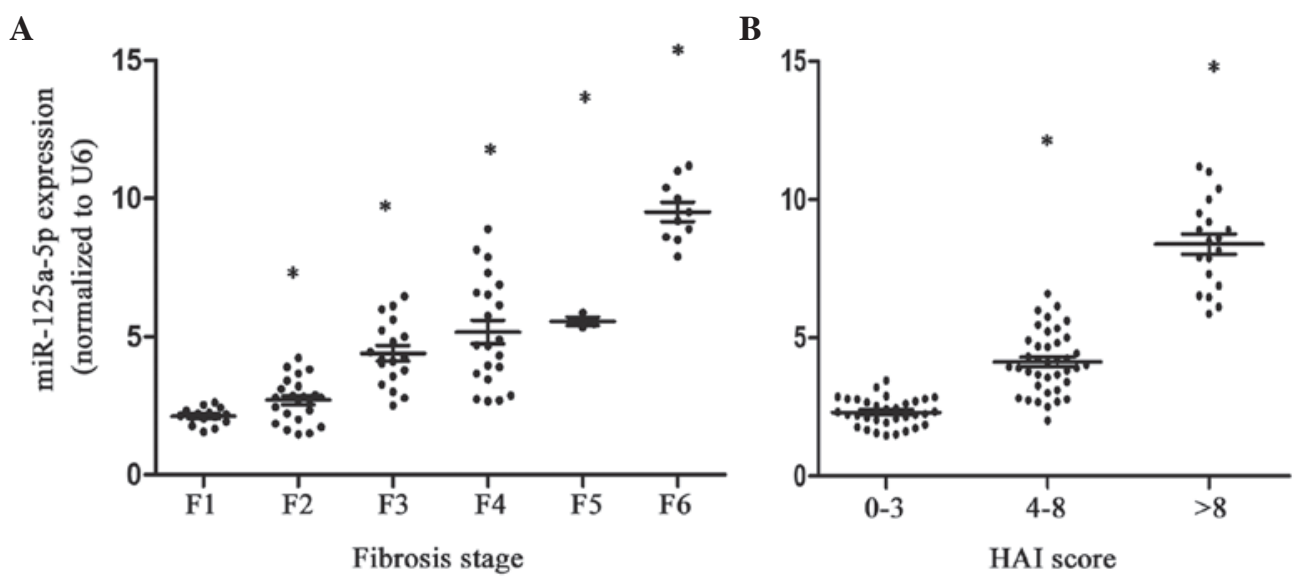

Figure 2. Scatter plots of miR-125a-5p levels at different fibrosis stages and HAI scores. ${ }^{*} \mathrm{P}<0.05$, compared with F1 or HAI 0-3. Data are presented as the median (range). F, stage of fibrosis; miR, microRNA; HAI, histological activity index.

$>2,000 \mathrm{IU} / \mathrm{ml}(19)$. miRNAs are important in regulating virus-host interactions (20). For example, with regards to HBV, miR-125a-5p can target a viral sequence within overlapping polymerase and surface antigen coding regions (21). Another previous study confirmed the effect of miR-125a-5p on the gee expression of HBV in the cultured hepatocytes (22). In 
Table III. Levels of hepatitis B virus DNA in the liver fibrosis group.

\begin{tabular}{|c|c|c|c|c|c|c|}
\hline \multirow[b]{2}{*}{ Group } & \multirow[b]{2}{*}{$\mathrm{n}$} & \multirow[b]{2}{*}{ Median } & \multicolumn{2}{|c|}{ Data range (IU/ml) } & \multicolumn{2}{|c|}{ Data range $(\mathrm{IU} / \mathrm{ml})$} \\
\hline & & & $5 \%$ & $95 \%$ & $5 \%$ & $95 \%$ \\
\hline Liver fibrosis & 91 & $3.40 \times 10^{4}$ & $5.50 \times 10^{3}$ & $3.90 \times 10^{6}$ & $1.46 \times 10^{3}$ & $5.00 \times 10^{8}$ \\
\hline $\mathrm{F} 1$ & 15 & $9.00 \times 10^{3}$ & $3.90 \times 10^{3}$ & $5.10 \times 10^{4}$ & $1.10 \times 10^{3}$ & $4.40 \times 10^{5}$ \\
\hline $\mathrm{F} 2$ & 24 & $2.60 \times 10^{4}$ & $6.95 \times 10^{3}$ & $3.90 \times 10^{5}$ & $1.12 \times 10^{3}$ & $2.83 \times 10^{7}$ \\
\hline F3 & 18 & $1.75 \times 10^{5}$ & $7.05 \times 10^{3}$ & $6.30 \times 10^{7}$ & $2.60 \times 10^{3}$ & $7.90 \times 10^{8}$ \\
\hline $\mathrm{F} 4$ & 21 & $6.80 \times 10^{3}$ & $3.45 \times 10^{3}$ & $5.20 \times 10^{6}$ & $1.45 \times 10^{3}$ & $6.00 \times 10^{8}$ \\
\hline F5 & 3 & $4.40 \times 10^{7}$ & $6.50 \times 10^{5}$ & $7.10 \times 10^{8}$ & $6.50 \times 10^{5}$ & $7.10 \times 10^{8}$ \\
\hline F6 & 10 & $3.65 \times 10^{6}$ & $6.80 \times 10^{4}$ & $3.71 \times 10^{7}$ & $6.00 \times 10^{3}$ & $8.10 \times 10^{8}$ \\
\hline
\end{tabular}

HCC, hepatocellular carcinoma; F, fibrosis stage.

Table IV. Expression levels of serum miR-125a-5p and other markers in the liver fibrosis group.

\begin{tabular}{lrrrrr}
\hline & & \multicolumn{2}{c}{ Data range $(\mu \mathrm{g} / \mathrm{l})$} & \multicolumn{2}{c}{ Data range $(\mu \mathrm{g} / \mathrm{l})$} \\
\cline { 3 - 4 } Marker & Median & $25 \%$ & $75 \%$ & $5 \%$ & $95 \%$ \\
\hline HA & 277.00 & 163.10 & 438.00 & 67.81 & 856.60 \\
LN & 85.20 & 66.65 & 135.50 & 24.83 & 191.70 \\
PCIII & 78.50 & 67.50 & 126.70 & 18.31 & 174.30 \\
IV-C & 82.40 & 45.45 & 114.40 & 17.50 & 158.40
\end{tabular}

HA, hyaluronic acid; LN, laminin; PCIII, type III procollagen protein; IV-C, type IV collagen; miR, microRNA.

A

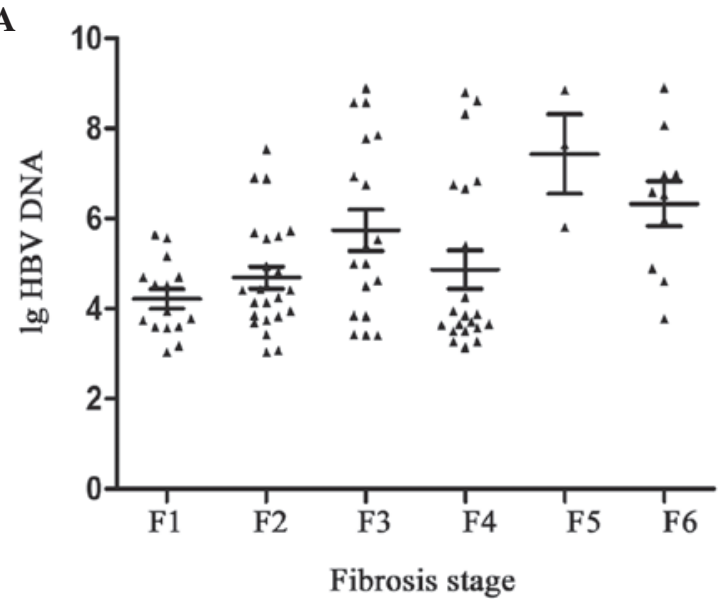

B

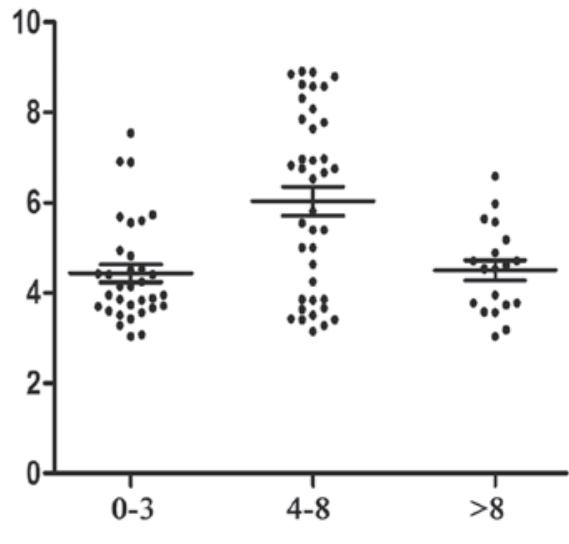

HAI score

Figure 3. Scatter plots of the levels of HBV DNA at different fibrosis stages and HAI scores. ${ }^{*} \mathrm{P}<0.05$, compared with F1 or HAI 0-3. Data are presented as the median (range). F, fibrosis stage; Ig, immunoglobulin; HBV, hepatitis B virus; HAI, histological activity index.

the present study, the correlation between serum miR-125a-5p and viral replication was analyzed. The results demonstrated that increased expression levels of serum miR-125a-5p were correlated with higher levels of HBV-DNA levels. This finding was similar to the correlation between the liver miR-125a-5p and HBV-DNA plasma levels (5).

Liver fibrosis is characterized by an excessive accumulation of extracellular matrices and is a common feature of chronic liver injury (23). Confirmation of the stages of liver fibrosis and the inflammatory grades is crucial for estimating disease progression, treatment selection and monitoring of the disease (24). With the exception of liver biopsy, the levels of serum HA, LN, PCIII and IV-C are often analyzed to estimate the degree of liver fibrosis, as fluctuation in their concentrations is consistent with the stages of liver fibrosis (25-27). In the present study, the concentrations of serum HA, LN, PCIII and IV-C were examined in the liver fibrosis group. Notably, the expression levels of serum miR-125a-5p were correlated 
Table V. Spearman's correlation coefficients of the correlation between serum miR-125a-5p and markers of liver fibrosis in the liver fibrosis group.

\begin{tabular}{lcr}
\hline Marker & r-value & P-value \\
\hline HA & 0.931 & $<0.001$ \\
LN & 0.211 & $<0.001$ \\
PCIII & 0.763 & $<0.001$ \\
IV-C & 0.728 & $<0.001$ \\
\hline
\end{tabular}

HA, hyaluronic acid; LN, laminin; PCIII, type III procollagen protein; IV-C, type IV collagen, miR, microRNA; r, Spearman's correlation coefficient.

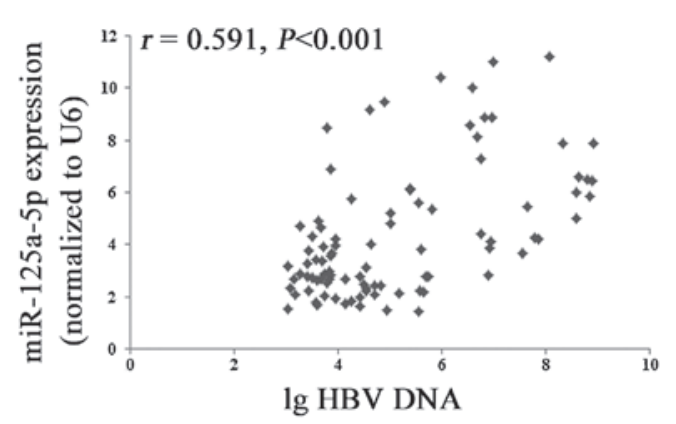

Figure 4. Association between the levels of serum miR-125a-5p and HBV DNA in patients with CHB. The significance was calculated according to Spearman's correlation. The $\mathrm{P}$ and $\mathrm{r}$ values are indicated. miR, microRNA; Ig, immunoglobulin; HBV, hepatitis B virus; r, correlation coefficient; CHB, chronic hepatitis $\mathrm{B}$.

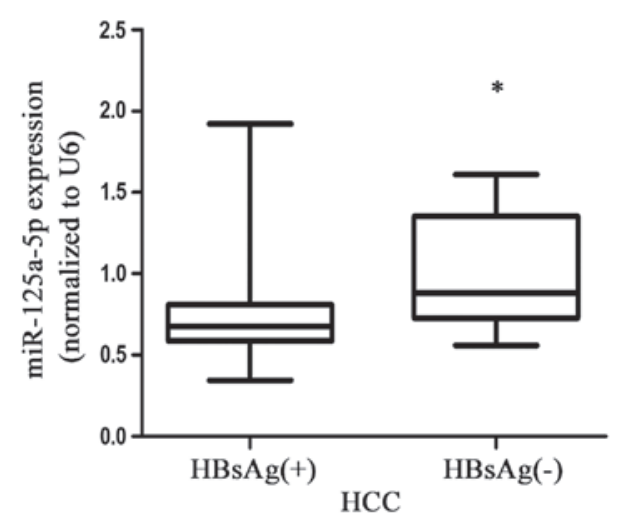

Figure 5. Levels of serum miR-125a-5p were detected in patients with HCC with HBsAg (+) or HBsAg (-). ${ }^{*} \mathrm{P}<0.05$, compared with HBsAg $(+)$. Data are presented as the median(range).miR, microRNA; HCC, hepatocellularcarcinoma.

with these markers, indicating that it may be used as a potential marker for liver fibrosis.

HCC accounts for $90 \%$ of the cases of primary liver cancer and represents the third most common cause of cancer-associated mortality worldwide (28). HCC is associated with hepatitis virus infection (29) and one of the major risk factors is HBV. Previous studies reported that serum microRNAs may be used as biomarkers for HCC (30). The present study demonstrated that serum miR-125a-5p was significantly reduced in the HCC group compared with the healthy control and liver fibrosis groups. In addition, higher

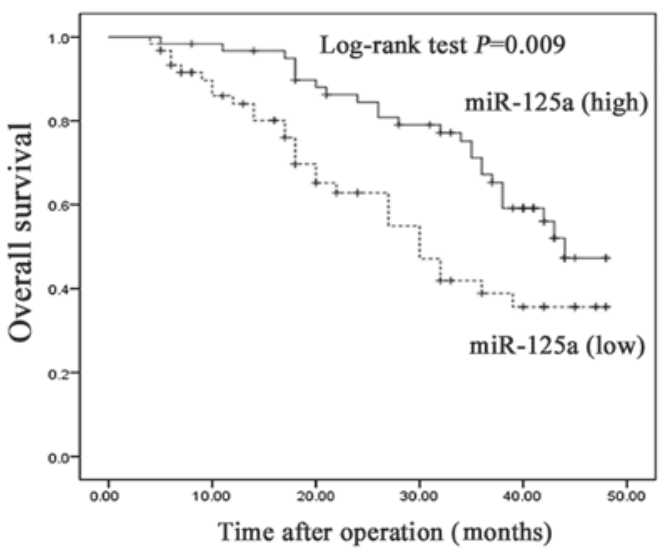

Figure 6. Overall survival rates of patients with HCC exhibiting high of low expression levels of serum miR-125a-5p were analyzed using the Kaplan-Meier method and log-rank test. miR, microRNA; HCC, hepatocellular carcinoma.

expression levels of serum miR-125a-5p were observed in the patients with $\mathrm{HBsAg}(+) \mathrm{HCC}$, indicating a correlation between serum miR-125a-5p and hepatitis virus infection. The present study also demonstrated that lower expression levels of serum miR-125a-5p were correlated with a poor prognosis, which was consistent with previous observations in tissue samples (4). These results indicated that serum miR-125a-5p may be used as a prognostic marker for HCC. In addition, Bi et al demonstrated that overexpression of miR-125a inhibits the proliferation and metastasis of HCC cells by targeting matrix metalloproteinase 11 and vascular endothelial growth factor A (4), indicating that miR-125a may be important as a suppressor of HCC proliferation and metastasis.

In the present study, fluctuations in the expression levels of miR-125a-5p in the serum were similar to those in liver tissue, indicating that serum miR-125a-5p may be released from the liver tissue, as the expression levels of serum miR-125a-5p were higher in the liver fibrosis group and lower in the HCC group. The levels of serum miR-125a-5p were significantly different in these two groups, indicating that serum miR-125a-5p may be a useful marker for liver diseases. However, the reason for the marked change in expression levels of serum miR-125a-5p in the liver fibrosis and HCC requires further investigation.

In conclusion, thee results of the present study demonstrated that the expression levels of serum miR-125a-5p differed in liver diseases, including liver fibrosis and HCC, 
and were correlated with disease progression. These results suggested that serum miR-125a-5p may be used as a marker for liver diseases.

\section{Acknowledgements}

The study was supported by the National Natural Science Foundation of China (nos. 81000176/H0317 and 81100292/H0317), the Zhejiang Provincial Natural Science Foundation of China (nos. Y2090326, Y2110634 and Y12H200010), the Department of Science and Technology of Zhejiang Province (no. 2013C37006) and the Wenzhou Municipal Science and Technology Bureau (no. Y20120127).

\section{References}

1. Ambros V: The functions of animal microRNAs. Nature 431: $350-355,2004$

2. Li L, Guo Z, Wang J, Mao Y and Gao Q: Serum miR-18a: a potential marker for hepatitis B virus-related hepatocellular carcinoma screening. Dig Dis Sci 57: 2910-2916, 2012.

3. Ding J, Huang S, Wu S, et al: Gain of miR-151 on chromosome 8q24.3 facilitates tumour cell migration and spreading through downregulating RhoGDIA. Nat Cell Biol 12: 390-399, 2010.

4. BiQ, Tang S, Xia L, et al: Ectopic expression of MiR-125a inhibits the proliferation and metastasis of hepatocellular carcinoma by targeting MMP11 and VEGF. PloS One 7: e40169, 2012.

5. Coppola N, Potenza N, Pisaturo M, et al: Liver microRNA hsa-miR-125a-5p in HBV chronic infection: correlation with HBV replication and disease progression. PLoS One 8: e65336, 2013.

6. Chen X, Ba Y, Ma L, et al: Characterization of microRNAs in serum: a novel class of biomarkers for diagnosis of cancer and other diseases. Cell Res 18: 997-1006, 2008.

7. Fang C, Zhu DX, Dong HJ, et al: Serum microRNAs are promising novel biomarkers for diffuse large B cell lymphoma. Annals Hematol 91: 553-559, 2012.

8. Gilad S, Meiri E, Yogev Y, et al: Serum microRNAs are promising novel biomarkers. PLoS One 3: e3148, 2008.

9. Mitchell PS, Parkin RK, Kroh EM, et al: Circulating microRNAs as stable blood-based markers for cancer detection. Proc Natl Acad Sci USA 105: 10513-10518, 2008.

10. Kim KH, Kim ND and Seong BL: Discovery and development of anti-HBV agents and their resistance. Molecules 15: 5878-5908, 2010.

11. Liu X, Wan X, Li Z, Lin C, Zhan Y and Lu X: Golgi protein 73 (GP73), a useful serum marker in liver diseases. Clin Chem Lab Med 49: 1311-1316, 2011.

12. Chen CJ, Yang HI, Su J, et al: Risk of hepatocellular carcinoma across a biological gradient of serum hepatitis B virus DNA level. JAMA 295: 65-73, 2006.
13. 2004 guidelines for surgical treatment of primary hepatocellular carcinoma. Chinese Journal of Hepatology 13: 329-330, 2005.

14. Ishak K, Baptista A, Bianchi L, et al: Histological grading and staging of chronic hepatitis. J Hepatol 22: 696-699, 1995.

15. Yao J, Liang L, Huang S, et al: MicroRNA-30d promotes tumor invasion and metastasis by targeting Galphai 2 in hepatocellular carcinoma. Hepatology 51: 846-856, 2010.

16. Lau DT and Bleibel W: Current status of antiviral therapy for hepatitis B. Therap Adv Gastroenterol 1: 61-75, 2008.

17. Bayram A, Erkilic S, Ozkur A, Bayram M and Sari I: Quantification of intrahepatic total hepatitis B virus DNA in chronic hepatitis B patients and its relationship with liver histology. J Clin Pathol 61: 338-342, 2008.

18. Yuen MF, Ng IO, Fan ST, et al: Significance of HBV DNA levels in liver histology of HBeAg and Anti-HBe positive patients with chronic hepatitis B. Am J Gastroenterol 99: 2032-2037, 2004.

19. European Association For The Study Of The Liver: EASL Clinical Practice Guidelines: management of chronic hepatitis B. J Hepatol 50: 227-242, 2009.

20. Russo A and Potenza N: Antiviral effects of human microRNAs and conservation of their target sites. FEBS Lett 585: 2551-2555, 2011.

21. Potenza N, Papa U, Mosca N, Zerbini F, Nobile V and Russo A: Human microRNA hsa-miR-125a-5p interferes with expression of hepatitis B virus surface antigen. Nucleic Acids Res 39: 5157-5163, 2011.

22. Park SO, Kumar M and Gupta S: TGF-beta and iron differently alter HBV replication in human hepatocytes through TGF-beta/ BMP signaling and cellular microRNA expression. PloS One 7: e39276, 2012.

23. Sekiya Y, Ogawa T, Yoshizato K, Ikeda K and Kawada N: Suppression of hepatic stellate cell activation by microRNA-29b. Biochem Biophys Res Commun 412: 74-79, 2011.

24. VizzuttiF, Arena U, Rega L and Pinzani M: Non invasive diagnosis of portal hypertension in cirrhotic patients. Gastroenterol Clin Biol 32: 80-87, 2008.

25. Xie SB, Yao JL, Zheng RQ, Peng XM and Gao ZL: Serum hyaluronic acid, procollagen type III and IV in histological diagnosis of liver fibrosis. Hepatobiliary Pancreat Dis Int 2: 69-72, 2003.

26. Parsian H, Rahimipour A, Nouri M, et al: Serum hyaluronic acid and laminin as biomarkers in liver fibrosis. J Gastrointestin Liver Dis 19: 169-174, 2010.

27. Lydatakis H, Hager IP, Kostadelou E, Mpousmpoulas S, Pappas S and Diamantis I: Non-invasive markers to predict the liver fibrosis in non-alcoholic fatty liver disease. Liver Int 26 : 864-871, 2006

28. Ahmed F, Perz JF, Kwong S, Jamison PM, Friedman C and Bell BP: National trends and disparities in the incidence of hepatocellular carcinoma, 1998-2003. Prev Chronic Dis 5: A74, 2008.

29. Parkin DM, Bray F, Ferlay J and Pisani P: Global cancer statistics, 2002. CA Cancer J Clin 55: 74-108, 2005.

30. Qi P, Cheng SQ, Wang H, Li N, Chen YF and Gao CF: Serum microRNAs as biomarkers for hepatocellular carcinoma in Chinese patients with chronic hepatitis B virus infection. PLoS One 6: e28486, 2011. 